\title{
Investigation of Titanium-Nitride Layers for Solar-Cell Contacts
}

\author{
HERMANN VON SEEFELD, NATHAN W. CHEUNG, MARTTI MÄENPÄÄ, AND MARC-A. NICOLET, MEMBER, IEEE
}

\begin{abstract}
Reactively sputtered titanium-nitride layers have been incorporated as diffusion barriers in a titanium-silver metallization scheme on silicon. Backscattering analysis $\left(2-\mathrm{MeV} \mathrm{He}^{+}, \mathrm{RBS}\right)$ indicates that the integrity of the system is basically preserved during annealing at $600^{\circ}$ for $10 \mathrm{~min}$. Electrical properties were determined for titaniumnitride layers prepared under different deposition conditions. Resistivity and Hall mobility appear to depend on the oxygen contamination of the deposited material. For the lowest oxygen concentration $(<5$ at $\%)$ a resistivity of $170 \mu \Omega \cdot \mathrm{cm}$ has been found.
\end{abstract}

\section{INTRODUCTION}

$\mathrm{T}$ ERRESTRIAL application of the photovoltaic technology demands low-cost and long-term reliable solar cells. Frequently, the failure of conventional cells is caused by the deterioration of electrical contacts near the $p-n$ junction. As pointed out by various authors [1],[2], the solderless $\mathrm{Ti}-\mathrm{Ag}$ metallization scheme will degrade when stored in a humid environment. Elevated temperature will accelerate the degradation. When subjected to a high temperature and humidity test $\left(90^{\circ} \mathrm{C}, 90-100\right.$ percent relative humidity), the cells were reported to have a reduced output and poor-adhesion qualities of the silver layer on titanium. To circumvent the problem, alternative metallization schemes have been proposed, such as the Ti-Pd-Ag system of Fischer and Gereth [3], or the allsilver front-contact scheme given by Hamneck et al. [4].

At present, the $\mathrm{Ti}-\mathrm{Pd}-\mathrm{Ag}$ system is employed by most solar cell manufacturers. Although the electrochemically passivated Ti-Pd-Ag combination has satisfactory electrical and mechanical properties after being subjected to a heat and humidity test, it is observed that the system undergoes structural changes during the exposure [3]. Modern packaging processes for solar cells also require an electrostatic bonding step, in particular the metallization scheme has to withstand a treatment at $600^{\circ} \mathrm{C}$ for $10 \mathrm{~min}$. At such elevated temperatures, very effective interdiffusion can take place at the metal-silicon interface. Consequently, the presence of metallic impurities near the $p-n$ junction can degrade the photovoltaic conversion mechanism. This problem is particularly pertinent to shallow-junction solar cells $(2000 \AA)$. An example of this kind of failure will be demonstrated in Section II.

It seems to be promising to apply the concept of diffusion barriers [5] to solar-cell metallization schemes, in order to

Manuscript received August 16, 1979; revised December 11, 1979. This research was supported initially by the Jet Propulsion Laboratory (R. Lutwack) and subsequently by the Department of Energy, monitored by Sandia Laboratories, Albuquerque, NM.

H. von Seefeld, N. Cheung, and M-A. Nicolet are with the California Institute of Technology, Pasadena, CA 91125.

M. Mäenpää is with the California Institute of Technology, Pasadena, CA 91125 , on leave from the Semiconductor Laboratory, Technical Research Center of Finland, SF-02150 Espoo 15, Finland. solve the contact problem. The idea is to interpose a material "X," the diffusion barrier, between two layers A and B, so that the undesirable thermal diffusion is suppressed while the electrical and mechanical properties of the A-X-B structure remain unaltered or at least equivalent to the $A-B$ metal combination.

An important aspect of the diffusion-barrier concept is to free the designer from various metallurgical constraints. Since the choice of metals is generally dictated by metallurgical and processing considerations, the application of an appropriate diffusion barrier may provide more variety to the selection of materials.

\section{Thermal Instability of the Ti-Pd-Ag System}

The performance of an actual solar cell has been checked after an exposure to $600^{\circ} \mathrm{C}$ for $10 \mathrm{~min}$. The specimen was provided by a commercial manufacturer. A degradation of the cell's performance characteristic is observed, in particular, both $V_{o c}$, the open-circuit voltage, and $I_{s c}$, the short-circuit current are significantly reduced.

It is suspected that the applied metallization scheme, in particular the Ti-Pd-Ag front-contact employed, may be responsible for these alterations. To check this point a Ti-Pd-Ag layer combination has been prepared by electron-beam evaporation on (100) silicon. Fig. 1 shows the backscattering spectrum of the system taken after deposition. When being annealed at $600^{\circ} \mathrm{C}$ for $5 \mathrm{~min}$, the spectrum changes significantly as demonstrated in Fig. 2. The titanium has interacted with the palladium film, as indicated by the reduction and the broadening of the titanium signal and its displacement toward higher backscattering energies, that is to say, towards the surface. Correspondingly, the height of the palladium signal is reduced and displaced inward, approaching the silicon interface.

This result correlates well with the observed failure of the solar cell after an identical heat treatment. The belief is that the $p-n$ junction region can either be contaminated or even short-circuited by the presence of metallic elements.

\section{Titanium-Nitride Diffusion BarRier}

The choice of an appropriate diffusion-barrier material is dictated by some mandatory characteristics for a functional metallization scheme. A reasonable electrical conductivity must be guaranteed and an adequate stability against chemical reactions must be assured with respect to the adjacent metal layers. In addition, a practicable preparation mode should be available.

The selection of a compound is indicated to account for the condition of chemical stability, provided a sufficiently inert 


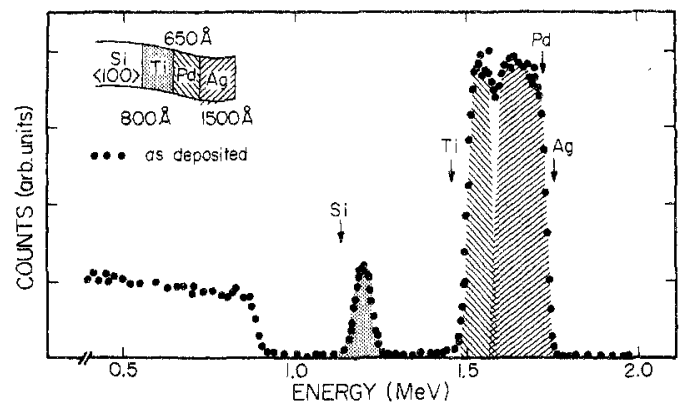

Fig. 1. Backscattering spectrum of $2-\mathrm{MeV}$ He ions obtained from a Ti-Pd-Ag metallization scheme on silicon. The spectrum was taken after deposition.

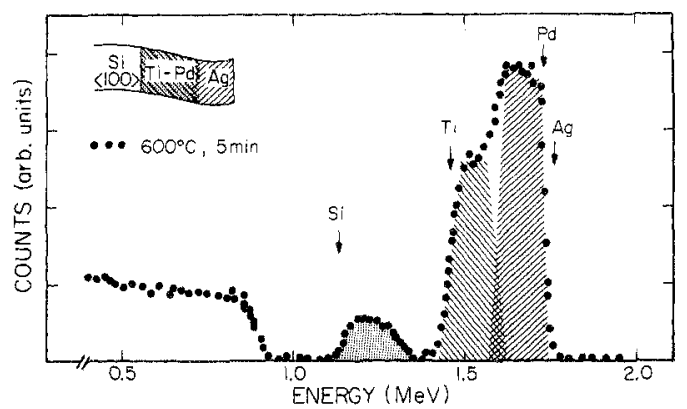

Fig. 2. Backscattering spectrum of $2-\mathrm{MeV}$ He ions obtained from a Ti$\mathrm{Pd}-\mathrm{Ag}$ metallization scheme on silicon after annealing at $600^{\circ} \mathrm{C}$ for $5 \mathrm{~min}$.

material can be found. Carbides, borides, and nitrides of the transition metals are promising choices due to their strong chemical bondings. Furthermore, they offer acceptable low bulk resistivities [5]. Titanium-nitride was chosen to replace palladium in the previously mentioned $\mathrm{Ti}-\mathrm{Ag}$ scheme. This decision has been made to avoid unnecessary complexity of the system. Furthermore, good adhesion is expected to result between the titanium and the nitride layer.

\section{Sample Preparation and Characterization, METHOdS OF MEASUREMENTS}

The metallization scheme actually investigated consists of a silicon single-crystal wafer, sequentially covered with a titanium, a titanium-nitride, and a silver layer: Si-Ti-TiN-Ag.

The metallic films were deposited by electron-beam evaporation. The nitride layer was prepared by reactive sputter. deposition. The sputtering process has been carried out in an RF-powered plasma-discharge assembly, using a titanium target and nitrogen as the sputtering gas to provide reactively formed titanium-nitride (TiN). Controlled deposition parameters are the gas pressure, the RF power, and the deposition time

Different test samples of the previously mentioned structure were prepared. The thicknesses of the metal layers were held constant, i.e., 3000- $\AA \mathrm{Ti}$ and $1300-\AA \mathrm{Ag}$. The samples differ essentially in the thickness and the preparation conditions of the nitride interlayer. The sputtering parameters, in particular the pressure and the power, were varied over a wide range. The deposition times were selected so as to obtain different layer thicknesses.
Analysis was carried out by backscattering technicue (2-JW6 V $\mathrm{He}^{+} \mathrm{RBS}$ ), and by X-ray diffraction. Backscattering analy permits the layer thicknesses and atomic composition to be measured; compare the Appendix and [6]. In addition, composition changes and depth distributions of migrating speciss (depth profiles) can be traced. To a certain extent, a quantitative detection of impurities is possible. X-ray diffraction provides information about the crystalline structure of the sample; in particular, phase formation can be confirmed.

Electrical properties of sputter-deposited nitride layers were obtained by van der Pauw's method [7] and by four-pointprobe measurements, using depositions on separate quartz samples. Sheet resistivities, sheet carrier concentrations, and Hall mobilities were evaluated. Layer thicknesses obtained from backscattering data permitted the corresponding bulk values to be deduced. The atomic composition of reactively prepared nitride turns out to be almost stoichiometric (TiN) with the tendency toward a slight excess of nitrogen. Oxygen is always present, but in general at concentrations only slightly above the quantitative determination limit, which is about 5 at $\%$ for the backscattering technique employed. Typically, the titanium-nitrogen-oxygen concentrations are found between the extreme values given below:

\begin{tabular}{ccc}
\hline $\mathrm{Ti}=1$ & $\mathrm{O} / \mathrm{Ti}$ & $\mathrm{N} / \mathrm{Ti}$ \\
\hline $\max :$ & 0.5 & 2.1 \\
$\min :$ & $\epsilon$ & 1.1
\end{tabular}

$\epsilon$ signifies an oxygen concentration lower than 5 at \%. X-ray diffraction patterns show that the nitride films have a facecentered-cubic polycrystalline structure. A TEM micrograph indicates grain sizes between 100 and $200 \AA$.

\section{EFfectiveness of TiN as a DIfFusion BarRier}

After preparation, the multilayered samples were subjected to an annealing at $600^{\circ} \mathrm{C}$ for $10 \mathrm{~min}$. A reference sample, consisting of the pure $\mathrm{Ti}-\mathrm{Ag}$ layer combination, was included for subsequent comparison. The annealing was carried out in a vacuum furnace at an approximate pressure of $5 \times 10^{-7}$ torr.

In case of the reference sample, a titanium-silver reaction is observed, leading to a compound of approximate atomic composition $\mathrm{AgTi}_{3}$. Annealed samples provided with a nitride interlayer exhibit a considerable reduction of the titaniumsilver interdiffusion. The spectra shown in Fig. 3 are taken before and after annealing. It is observed that the integrity of the system is basically preserved during the heat treatment but a limited amount of titanium is detected at the surface. The presence of some titanium at the surface is a general observation for all samples considered. The suppression of the titanium diffusion is observed to be more effective if thicker nitride layers are chosen. No particular dependence of the barrier action on the deposition parameters "power" and "pressure" could be detected.

Since no titanium-silver compound formation is observed if the nitride layer is present, it is suspected that grain boundary diffusion accounts for the titanium migration to the surface. Another explanation of the spectrum observed would be the 


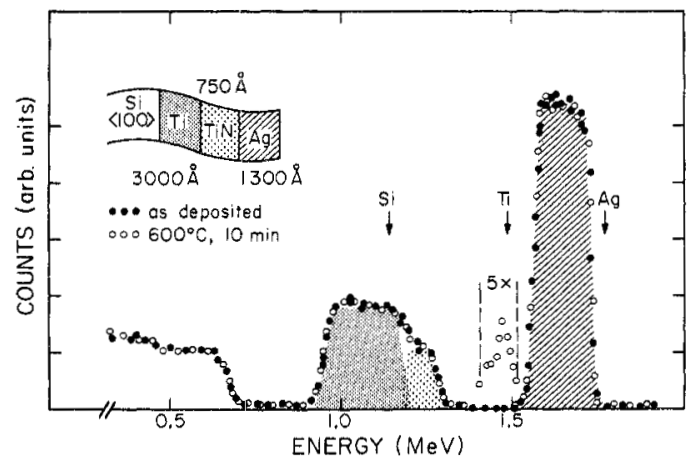

Fig. 3. Backscattering spectra $\left(2-\mathrm{MeV} \mathrm{He}{ }^{+}\right)$of a Ti-TiN-Ag metallization scheme on silicon, taken before $(\bullet \bullet \bullet)$ and after $\left(\begin{array}{llll}0 & \circ & 0\end{array}\right)$ annealing at $600^{\circ} \mathrm{C}$ for $10 \mathrm{~min}$.

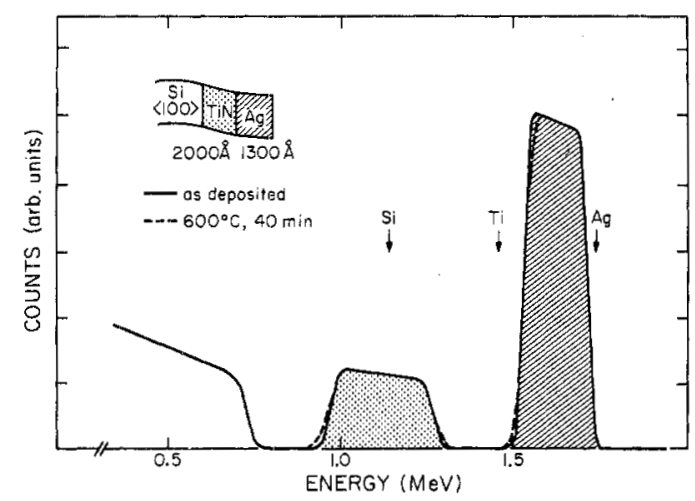

Fig. 4. Backscattering spectra $\left(2-\mathrm{MeV} \mathrm{He}{ }^{+}\right)$of a $\mathrm{TiN}-\mathrm{Ag}$ metallization scheme on silicon, taken before (-) and after (-- - ) annealing at $600^{\circ} \mathrm{C}$ for $40 \mathrm{~min}$.

formation of lateral nonuniformities in the silver layer (e.g., pinholes and microcracks) during the annealing step.

Fig. 4 shows the backscattering spectra of a different test sample. Titanium-nitride was applied directly to the silicon wafer, covered with a similar silver overlayer. A heat treatment at $600^{\circ} \mathrm{C}$ up to $40 \mathrm{~min}$ reveals almost no changes in the sample's composition.

\section{Electrical Properties}

Electrical properties of titanium-nitride layers were investigated with particular attention to their dependence on the sputtering conditions and on the atomic composition of the resulting films. Measured resistivities vary between 170 and $1050 \mu \Omega \cdot \mathrm{cm}$. These values correlate with the Hall mobilities obtained ranging from 0.88 to $0.16 \mathrm{~cm}^{2} / \mathrm{V} \cdot \mathrm{s}$, respectively. According to Hall measurements, the charge carriers are electrons. With reference to film thicknesses obtained by backscattering analysis, a carrier concentration of about $4 \times 10^{22}$ $\mathrm{cm}^{-3}$ is deduced in all cases.

The oxygen concentration of the layer appears to be a most influential parameter with respect to electrical characterization. Fig. 5 depicts the resistivity, the electron concentration, and the Hall mobility, each as a function of the oxygen-titanium concentration ratio. The lowest resistivities and, correspondingly, the highest mobilities result when the oxygen concentration is lower than the quantitative detection limit.

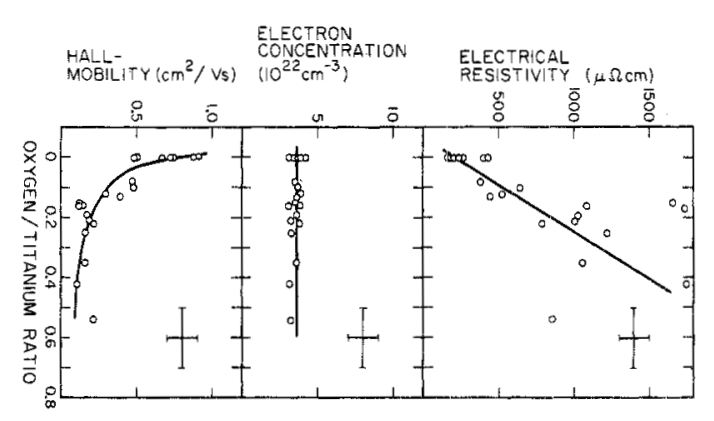

Fig. 5. Electrical resistivity, electron concentration, and Hall mobility of titanium-nitride layers, each as a function of the oxygen-titanium ratio.

Although a clear dependence of the resistivity on the film thickness could not be established, it has been observed in general that the oxygen concentration is higher as the layer thickness is reduced. A change of the nitrogen-titanium ratio does not affect the electrical properties. Furthermore, no correlation was found between the nitrogen-titanium ratio and the oxygen concentration.

\section{CONCLUSIONS}

Reactively sputtered titanium-nitride, when introduced as a separating layer in a $\mathrm{Ti}-\mathrm{Ag}$ metallization scheme, is shown to act as an effective barrier against interdiffusion and compound formation of the adjacent metals. When subjected to a heat treatment typical for solar-cell processing, an improvement of the system's stability is observed, compared with a conventional Ti-Pd-Ag scheme. Suppression of the Ti layer, i.e., considering the single $\mathrm{TiN}-\mathrm{Ag}$ layer combination, appears to be a promising alternative, too.

The electrical resistivity of a titanium-nitride layer is found to be sufficiently low to compete with pure metal contacts. Resistivity is observed to depend strongly on the oxygen concentration of the nitride. The highest conductivities result when the oxygen concentration is lower than our quantitative detection limit.

A definite correlation between the sputtering conditions and the composition of the resulting layer could not be established. Nevertheless, it is suspected that the sputtering parameters are decisive in determining film characteristics. Improved properties are expected to result from a judicious choice of the preparation conditions.

In summary, the application of titanium-nitride in silicon metallization schemes may provide a simple and low-cost alternative to existing solar-cell contact systems.

\section{APPENDIX}

This Appendix briefly describes the connection that exists between the structures of samples such as are presently considered and their backscattering spectra. A detailed treatment of the subject is found in the literature [6] .

The sample to be analyzed-also referred to as the target-is irradiated with high-energy particles, i.e., 2-MeV helium ions. A small fraction of the incoming ions experience large-angle deflections when interacting with a target atom and can be monitored by a detector placed in front of the specimen. De- 


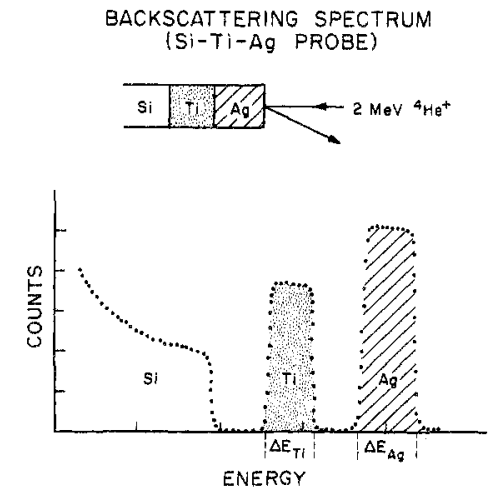

Fig. 6. Backscattering spectrum of a titanium-silver double-layer deposit on single crystalline silicon.

tected ions are counted and classified according to their energy. A representation of the number of detected ions (counts) versus energy is called a backscattering spectrum. The information deduced is considered to be an average over the irradiate area which is typically $1 \mathrm{~mm}$ in diameter.

Provided a monoenergetic primary beam is used, the energy of a scattered ion is essentially determined by the mass of the scattering atom and by the depth at which the collision occurred. The height of a spectrum at a particular energy (counts per energy interval) is a function of the atomic density of the scattering species. Consequently, a backscattering spectrum provides information about the masses and the densities of atomic species in the target and about the depth distribution of the latter. If an actual target structure is considered, for example, two thin films of titanium and silver deposited on a silicon substrate, the corresponding backscattering spectrum has the appearance shown in Fig. 6. Ions scattered by the silver layer are detected in an interval $\Delta E(\mathrm{Ag})$ located at high backscattering energies. Ions penetrating this layer and being scattered by the titanium atoms fall in an energy range $\Delta E(\mathrm{Ti})$ at intermediate energies. The substrate $(\mathrm{Si})$ contributes a continuous distribution covering the low-energy range of the spectrum.

The width of a particular interval $(\Delta E(\mathrm{Ag}), \Delta E(\mathrm{Ti}))$ is related to the thickness of the corresponding layer. A conversion of energy differences into length units is possible without reference to standards (depth scaling). As a rule of thumb, it may be stated that for an individual layer the energy difference and the thickness are approximately proportional, as the conversion factor is a function of the material's density. The signal amplitude for the silver layer is largest, and for the silicon layer is lowest, because the sensitivity of the technique increases for heavier elements.

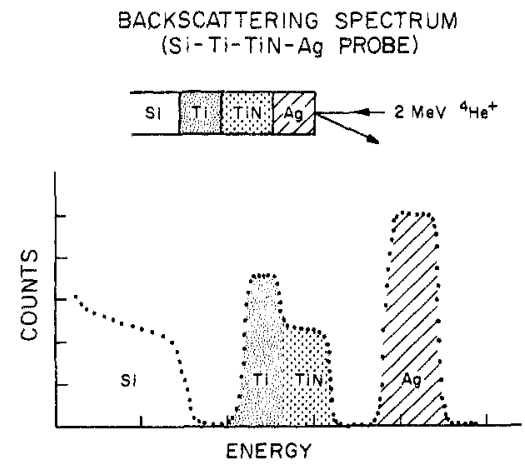

Fig. 7. Backscattering spectrum of a multilayered silicon metallization scheme, consisting in a 100 silicon crystal sequentialy covered with titanium, titanium-nitride, and silver.

Another example of interest is the spectrum of a sample including a titanium and a titanium-nitride layer, as shown in Fig. 7.

Concentrating on the stepped signal at intermediate energies, it states that in both, the nitride and the metal, titanium is the scattering species. The reduced yield corresponding to the nitride layer reflects the fact that the density of titanium atoms in the nitride is lower than in the pure metal.

The signal of the nitrogen in the nitride layer is small because nitrogen is a very light element. It is, consequently, difficult to detect, and it is omitted in the diagram.

\section{ACKNOWLEDGMENT}

The authors wish to thank $P$. Iles of Optical Coating Laboratory, Inc. for providing the solar cell wafers, $T$. Cullis of the Royal Signals and Radar Establishment for TEM results, and D. Fitzgerald of the Jet Propulsion Laboratory for helpful discussions.

\section{REFERENCES}

[1] W. H. Becker and S. R. Pollack, "The formations and degradation of $\mathrm{Ti}-\mathrm{Ag}$ and $\mathrm{Ti}-\mathrm{Pd}-\mathrm{Ag}$ solar cell contacts," in Proc. 8th IEEE Photovoltaic Specialists Conf. (Seattle, WA), p. 40, 1970.

[2] C. J. Bishop, "The fundamental mechanism of humidity degradation in silver-titanium contacts," in Proc. 8th IEEE Photovoltaic Specialists Conf. (Seattle, WA), p. 51, 1970.

[3] H. Fischer and R. Gereth, "Electrochemically passivated contacts for silicon solar cells," IEEE Trans. Electron Devices, vol. ED-10, no. 6, p. 459,1971 .

[4] J. H. Hamneck, Jr., L. Schwartz, and A. E. Spakowsk, "A reliable all-silver front contact for silicon solar cells," in Proc. 9th IEEE Photovoltaic Specialists Conf. (Silver Spring, MD), p. 193, 1972.

[5] M-A. Nicolet, "Diffusion barriers in thin films," Thin Solid Films, vol. 52 , pp. $415-443,1978$.

[6] W. K. Chu, M-A Nicolet, and J. W.-Mayer, Backscattering Spectrometry. New York: Academic Press, 1978.

[7] L. J. van der Pauw, Philips Tech. Rundsch., vol. 20, p. 230, (1958/ 1959). 